NASA Technical Memorandum 106951

\title{
Optimization of Wave Rotors for Use as Gas Turbine Engine Topping Cycles
}

Jack Wilson

NYMA, Inc.

Engineering Services Division

Brook Park, Ohio

and

Daniel E. Paxson

National Aeronautics and Space Administration

Lewis Research Center

Cleveland, Ohio

Prepared for the

1995 Aerospace Atlantic Conference and Exposition sponsored by the Society of Automotive Engineers

Dayton, Ohio, May 23-25, 1995

National Aeronautics and

Space Administration 


\title{
Optimization of Wave Rotors for Use as Gas Turbine Engine Topping Cycles
}

\author{
Jack Wilson \\ NYMA, Inc. \\ Brook Park, Ohio 44142 \\ Daniel E. Paxson \\ National Aeronautics and Space Administration \\ Lewis Research Center \\ Cleveland, Ohio 44135
}

\section{ABSTRACT}

Use of a wave rotor as a topping cycle for a gas turbine engine can improve specific power and reduce specific fuel consumption. Maximum improvement requires the wave rotor to be optimized for best performance at the mass flow of the engine. The optimization is a trade-off between losses due to friction and passage opening time, and rotational effects. An experimentally validated, one-dimensional CFD code, which includes these effects, has been used to calculate wave rotor performance, and find the optimum configuration. The technique is described, and results given for wave rotors sized for engines with sea level mass flows of 4,26 , and $400 \mathrm{lb} / \mathrm{sec}$.

\section{INTRODUCTION}

Wave rotors are devices which use unsteady waves to compress and expand air in a single device. Basically, a wave rotor consists of a rotating bank of passages, inside a housing, with ports bringing air in, or taking air out. A four port wave rotor is shown in figure 1. Four port wave rotors have been tested by General Electric [1], and Rolls -Royce [2]. Other arrangements are possible [3]; however, the four port scheme is more attractive because it fits easily into a conventional engine. Consequently, only the four port scheme will be considered here. With a wave rotor installed as a topping cycle in a gas turbine engine, the air from the engine compressor would be directed into the wave rotor at the input port 1. (see figure 1). The air flows into the passages on the rotor, and is compressed by a series of compression or shock waves. This air leaves the wave rotor from port 2, at a higher pressure than when it entered the wave rotor, and passes to the burner. After being heated in the burner, the gas returns to the wave rotor through port 3 , driving a shock into the air in the passages in the process. This gas is trapped on board as port 3 closes, at quite high pressure. When the rotor comes around to the exit port 4 , the gas expands out into the lower static pressure in the port. From there the gas goes to the engine turbine. In passing through the wave rotor, the air is first compressed and then expanded. Thus the wave rotor combines in a single device the functions performed by the compressor and turbine in a high spool. By using a wave rotor topping cycle, combustion temperatures greater than the turbine inlet temperature can be used, since the gas leaving the combustor is

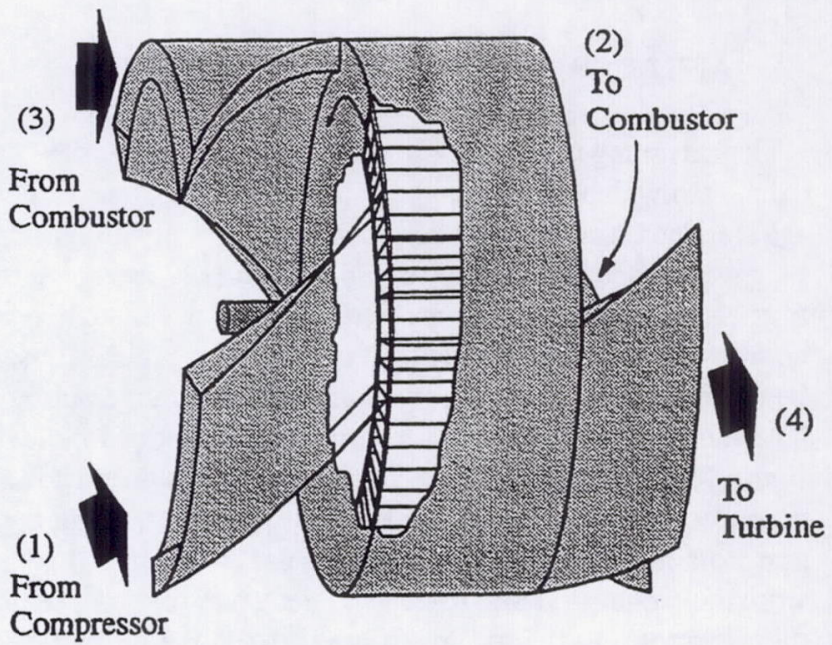

Figure 1 Schematic of a four port wave rotor

cooled in the expansion before being sent to the turbine. Also, since the rotor is washed alternately by cool inlet air and hot combustion gas, it is self-cooled, and attains a steady state temperature significantly lower than the combustion temperature. By increasing the overall cycle pressure ratio, and allowing higher combustion temperatures, the wave rotor topping cycle offers a potential route to higher engine efficiency.

The successful application of a wave rotor topping cycle is dependant upon how much performance improvement it can achieve over a conventional cycle. Calculations of improvements in specific power and efficiency have been reported [4]. In making such calculations, it is necessary to know the performance of the wave rotor. What is meant by wave rotor performance is the pressure gain created by the wave rotor as a function of the temperature ratio across it, i.e. $P_{4} / P_{1}$ versus $T_{4} / T_{1}$. Note that this pressure gain, $\mathrm{P}_{4} / \mathrm{P}_{1}$, is equivalent to the pressure at the exit of a turbine divided by the pressure at the entrance to the compressor. It should not be confused with the compressor pressure ratio, which is commonly used as a measure of performance in gas turbine engine cycles. In [4], the wave rotor pressure gain was taken to be that measured by Klapproth et al.[1]. This is a conservative assumption, in that this performance has clearly been demonstrated, but has certainly not been optimized. The better the performance of the wave rotor, 
the greater will be the increase in specific power, and reduction in specific fuel consumption of the engine/wave-rotor combination. The wave rotor performance will depend on the losses, which in turn depend on the geometry, and inlet conditions, of the wave rotor. What is needed then, is a realistic calculation of wave rotor performance, including the major losses, which can be used to examine variations in geometry to find optimum performance. A suitable, one-dimensional, computational fluid dynamics (CFD) code has been written $[5,6]$, and was used for this report. The code has been validated against experiment [7], and gives good agreement with the experimental results of Klapproth et al. [1], and Kentfield [8]. Using this code, an optimum wave rotor size has been found for hypothetical engines with mass flows typical of small, medium, and large gas turbine engines. Finally, the specific power and specific fuel consumption of these engines was calculated assuming a wave rotor topping cycle employing the optimized wave rotor, for comparison with the basic engine.

\section{ADVANTAGES OF USING A WAVE ROTOR}

The advantages of using a wave rotor topping cycle can be calculated with a simple model [4]. The details of the calculation are standard, and will not be repeated here; only an outline will be given. Because in the topping stage (i.e. the wave rotor burner combination) the gas is heated in a constant pressure combustor, thermodynamically it is a Brayton cycle. Thus, it can be represented on a temperature-entropy diagram as in figure 2 . The numbers in figure 2 correspond to the stations indicated in figure 1, i.e, 0 is the entrance to the engine, 1 is the exit from the compressor, and entry to the wave rotor, 2 is the high pressure exit from the wave rotor, and entry to the combustor, 3 is the exit from the combustor, and re-entry to the wave rotor, 4 is exit from the wave rotor to the turbine, 5 is exit from the turbine, and 6 is return to ambient pressure. The basic engine, to which the wave rotor is added, is a simple gas turbine engine, so that the work of compression is equal to the turbine work (except for mechanical losses ), and the available energy is simply the difference between the energy of expansion from a pressure $P_{1}$, and temperature $\mathrm{T}_{4}$ at turbine inlet to ambient conditions (at pressure $\mathrm{P}_{0}$ ), minus the turbine work. With a wave rotor topping stage added, because the stage acts as a pressure gain combustor, the

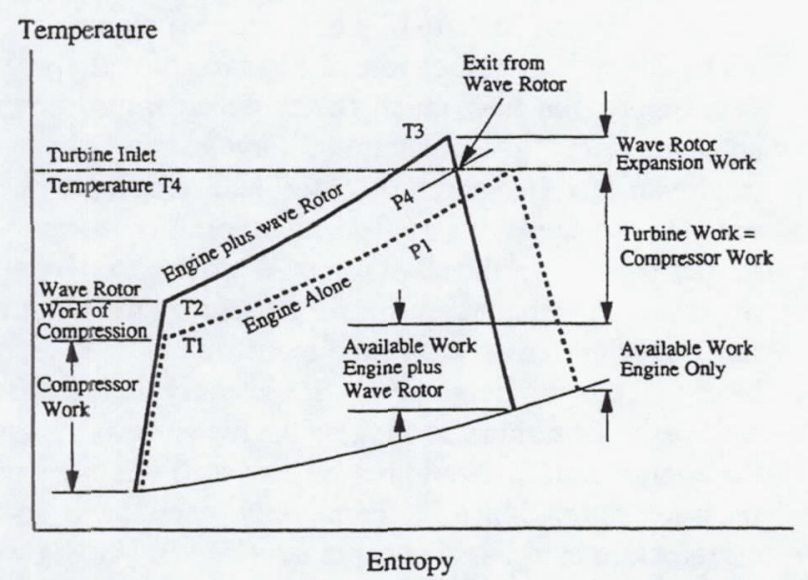

Figure 2 Temperature-entropy diagram for a gas turbine engine, and for a gas turbine engine with a wave rotor topping cycle

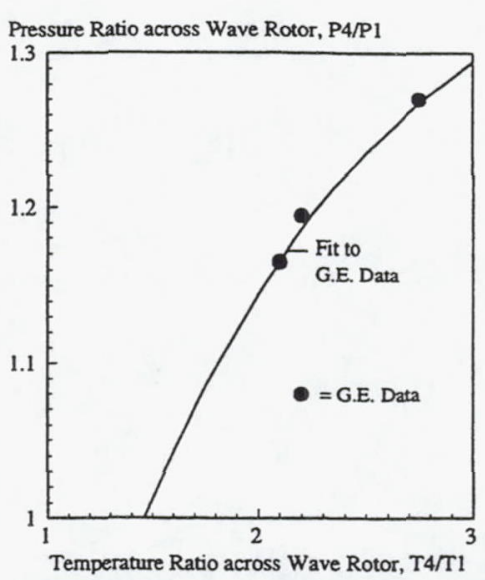

Figure 3 Wave rotor performance measured experimentally by Klapproth et al. [1], (), together with a curve-fit calculated with the CFD code $(-)$

pressure $\mathrm{P}_{4}$ leaving the wave rotor and entering the turbine is higher than the pressure $P_{1}$ entering the turbine in the case of the basic engine alone. Consequently the expansion between turbine inlet and ambient conditions is through a higher pressure ratio, and there is more available work.

It will be assumed that for the basic engine, the value of the compression ratio $\Pi_{s}=P_{1} / P_{0}$ is known, as well as the turbine inlet temperature, and the compression and turbine efficiencies. With these quantities given, the specific power and specific fuel consumption of the basic engine can be calculated. When a wave rotor topping stage is added, it will be assumed that the turbine inlet temperature remains unchanged, but the pressure entering the turbine is raised from $P_{1}$ to $P_{4}$. Thus to calculate the performance in this case, it is necesssary to know the pressure ratio $\mathrm{P}_{4} / \mathrm{P}_{1}$ generated by the wave rotor for a given temperature ratio across it, i.e. $T_{4} / T_{1}$.

The wave rotor pressure ratio as a function of the wave rotor temperature ratio is not a unique function. It depends on the geometry of the wave rotor, which will change with the mass flow of the engine. In [4] a fit to the results of Klapproth et al. [1] was used. As explained above, use of this fit is conservative; the performance should be amenable to improvement, since the experimental rotor was neither well timed, nor optimized. Moreover, the experimental rotor had a small mass flow; in general wave rotor performance improves as the mass flow increases. The curve-fit to the experimental results was obtained using the one-dimensional CFD code described below, with estimates of the geometry of the G.E. rotor, to calculate the wave rotor pressure ratio at different values of the wave rotor temperature ratio. The fitted curve is the line shown in figure 3. Also shown are the experimental points from [1], which are the solid circles. Although the fit to the experimental points appears to be quite good, this must be regarded as somewhat fortuitous. The timing (i.e. when the ports open and close) of the experimental rotor was not known, neither was the exact geometry, nor the size of any leakage gaps. Consequently an exact calculation was not possible. Nevertheless, the fit is obviously of the right magnitude, and should provide a good estimate of the wave rotor performance for this rotor. 


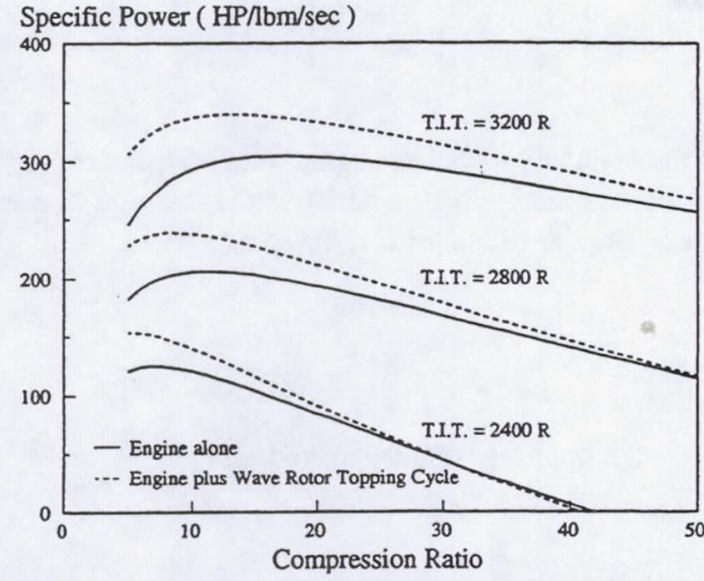

Figure 4 Specific power vs. shaft compression ratio for engines with and without a wave rotor topping cycle. The engines are assumed to have performance as calculated with the fit to the data of Klapproth et al. [1]

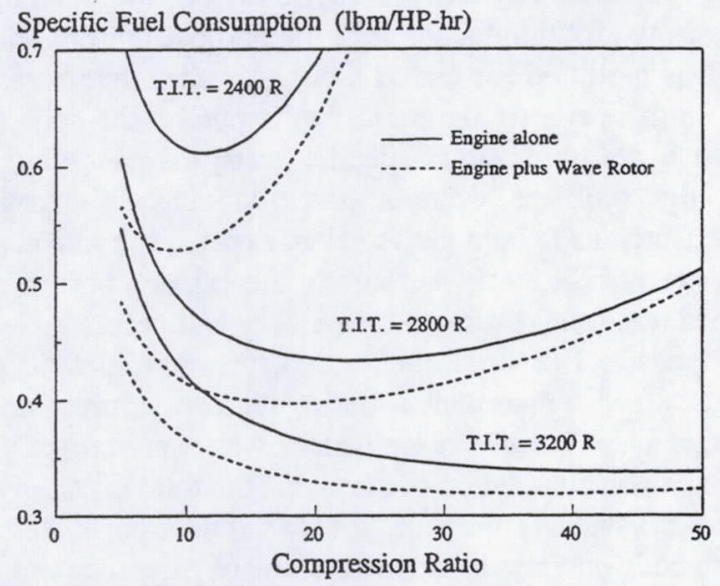

Figure 5 Specific fuel consumption vs. shaft compression ratio for engines with, and without a wave rotor topping cycle. The wave rotors are assumed to have performance as calculated with the fit to the data of Klapproth et al. [1]

With the wave rotor pressure ratio known from figure 3, the specific power, thermal efficiency, and specific fuel consumption can be calculated. In [4], calculations of the thermal efficiency of an untopped engine, calculated as above, were compared with results from the ONX program [9], and found to give excellent agreement, giving confidence in this approach.

With this model, and the curve-fit to the G.E. data, values of specific power and specific fuel consumption have been calculated for shaft compression ratios (i.e., the compression ratio of the mechanical compressor) between 5 and 60 , for engines with, and without, a wave rotor topping cycle. Turbine inlet temperatures of $2400 \mathrm{R}$, typical of a small engine, $2800 \mathrm{R}$, and $3200 \mathrm{R}$, appropriate for an advanced engine, were used. Sea level ambient conditions were assumed. The results are shown in figures 4 and 5 . It can be seen that quite large increases in specific power are possible by the addition of a wave rotor topping cycle, particularly at low values of the shaft compression ratio. For example, at a turbine inlet temperature of $2400 \mathrm{R}$, and a shaft compression ratio of 8 , addition of a wave rotor topping

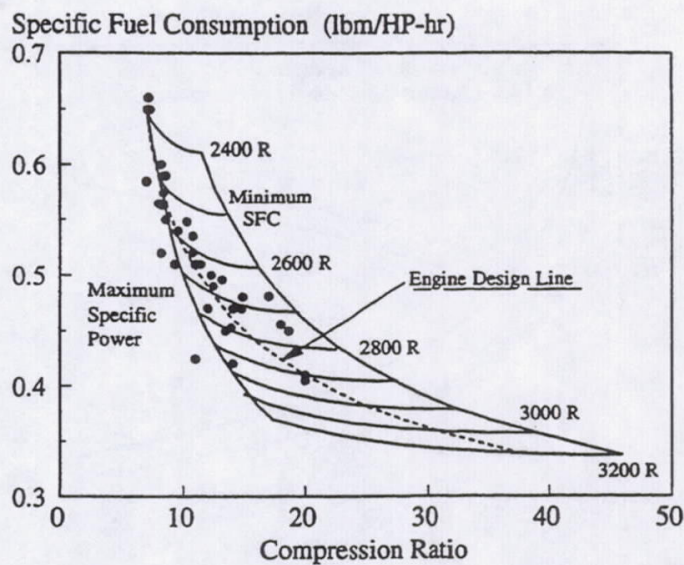

Figure 6 Specific fuel consumption vs. shaft compression ratio with turbine inlet temperature as a parameter

cycle increases the specific power by $19.2 \%$, and reduces the specific fuel consumption by $16.2 \%$. Raising the turbine inlet temperature to $3200 \mathrm{R}$ at a compression ratio of 8 reduces the gain. At these conditions, the specific power increases $17.4 \%$, and the specific fuel consumption decreases by $14.8 \%$. Increasing the compression ratio reduces the gain even more dramatically. At a shaft compression ratio of 40 , the specific power increase is $5.8 \%$, and the specific fuel consumption decrease is $5.6 \%$. An engine with a turbine inlet temperature of $2400 \mathrm{R}$, and a compression ratio of 40 would actually decrease its performance by the addition of a wave rotor topping cycle. However, neither an engine with a shaft compression ratio of 8 , and a turbine inlet temperature of $3200 \mathrm{R}$, nor an engine with a shaft compression ratio of 40 , and a turbine inlet temperature of $2400 \mathrm{R}$ are well designed engines; an actual engine is more likely to have a compression ratio somewhere between the value giving the minimum specific fuel consumption, and the value giving the maximum specific power for the turbine inlet temperature of that particular engine. The actual value is strong influenced by the mission requirements of the engine.

In figure 6, the specific fuel consumption of gas turbine engines without wave rotor topping cycles, as calculated with the simple model described above is plotted against compression ratio, with turbine inlet temperature as a parameter, for values of compression ratio between those for maximum specific power, and those for minimum specific fuel consumption. Also plotted are points (i.e. specific fuel consumption and compression ratio) for actual engines taken from [10]. In addition, there is a line, called the engine design line, which is an approximately average line through the data, which will be taken as an appropriate line relating compression ratio and turbine inlet temperature for an "average" engine. Thus for example, an engine with a compression ratio of 20 might be expected to have a turbine inlet temperature of $2900 \mathrm{R}$. Using this relationship between engine compression ratio and turbine inlet temperature, the specific power and specific fuel consumption were calculated for engines with, and without, a wave rotor topping cycle, and the results are plotted in figure 7. Again, at low values of shaft compression ratio, there is a significant gain; for a shaft compression ratio of 8 , the addition of a wave rotor topping cycle increases the specific power by $19 \%$, and reduces the specific fuel 


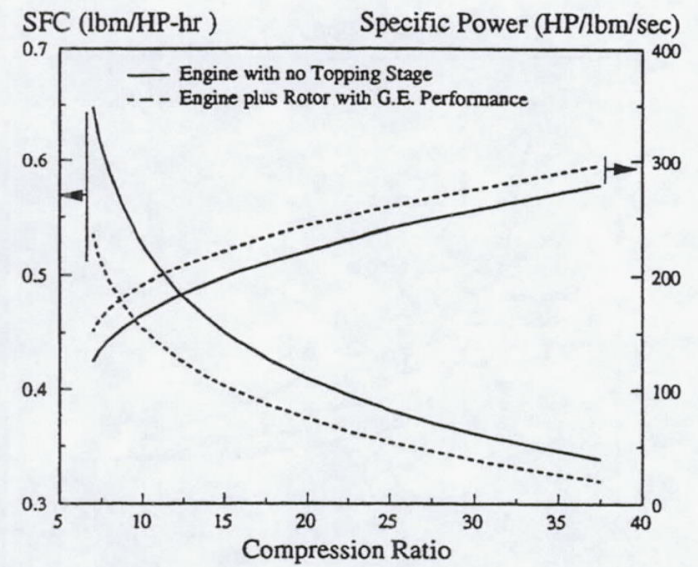

Figure 7 Specific power and specific fuel consumption for engines with, and without a wave rotor topping cycle. At any compression ratio, the basic engines are assumed to have turbine inlet temperatures corresponding to the design line of figure 6

consumption by $16.2 \%$. At higher values of shaft compression ratio, the advantage is lessened; for a shaft compression ratio of 40 , addition of a wave rotor topping cycle increases specific power by $5.8 \%$, and reduces specific fuel consumption by $5.6 \%$. It is worth re-emphasizing that the above results were derived using the wave rotor performance found experimentally [1]. This performance has been demonstrated, and therefore it should be possible to obtain the calculated increases in specific power, and decreases in specific fuel consumption. However, the experimental rotor used in [1] was not optimized even for the experimental mass flow, and is certainly not optimum for any engine. It will be shown below that wave rotor performance can be optimized for a given mass flow, and the resulting performance will be better than that demonstrated by Klapproth et al.[1]. Consequently the increase in specific power, and reduction in specific fuel consumption that can be obtained by the use of an optimized wave rotor topping cycle will also be greater. In order to perform this optimization, a model that can calculate wave rotor performance is required. Such a model is described in the next section.

\section{CFD WAVE ROTOR MODEL}

The computational wave rotor model is a one dimensional code which follows a single wave rotor passage as it rotates past the various ports of the cycle. The working fluid is assumed to be a calorically perfect gas. The details of this code have been described in previous publications [5-7] and will not be presented here. Although several loss mechanisms are accounted for, those of interest here result from the effects of viscosity, gradual passage opening (so called finite opening time), and mixing of non-uniform velocity profiles in the exhaust port 4 . For reference, viscous effects are accounted for in the model with a source term in the governing momentum equation. The strength of the source term is determined by local velocity, density and a constant friction factor which depends on the passage geometry. It is defined as

$$
f=5.448\left(\frac{L}{D_{h}}\right)^{1.081}\left(\frac{\rho^{*} a^{*} L}{\mu}\right)^{-.3953}
$$

Since the viscosity does not change much with temperature it is assumed constant at a value which is the average of ports 1 and 4. The hydraulic diameter is defined as

$$
D_{b}=2 \frac{b h}{b+h}
$$

The passage opening time is defined as

$$
\tau_{\mathrm{ot}}=\frac{\mathrm{ba}^{*}}{\mathrm{LR} \omega}
$$

Increases in either finite opening time or friction factor result in decreased wave rotor rotor performance. Furthermore, examination of EQS (1), (2) and (3) reveals that, aside from an increase in rotor angular velocity, reducing the opening time increases the friction factor. For the purposes of this study the code was modified for use as a design rather than an analysis tool. In this capacity the prescribed inputs to the code are the friction factor, the ratio of specific heats, the passage opening time, the ratio of exhaust port to inlet port stagnation temperature, $T_{4} / T_{1}$, and the so called expansion ratio, $\epsilon$, which is the ratio of the static pressure in the exhaust port, $p_{4}$, to the assumed uniform pressure in the passage just before the exhaust port opens. The code then calculates the inlet stagnation pressures and temperatures, outlet static pressures, and port locations which insure that the various waves are properly timed and that, upon completion of the cycle, the total mass flow from outlet ports matches the total flow to the inlet ports. It is noted that the code operates in the rotor frame of reference and that as such, all stagnation properties discussed in this section of the paper are to be understood as rotor relative. The balancing of mass flow also insures that the passage completes the cycle in nearly the same state at which it began so that the cycle may start again. The calculation process is an iterative one with several sub-processes. These are shown in flowchart form in Appendix A and described briefly below. A complete calculated cycle, along with the port nomenclature, is shown in figure 8 . This plot is a passage pressure contour with time (or circumferential position) and distance along the passage as the vertical and horizontal axes respectively. It is noted that although the design code can calculate the appropriate port timing and relative stagnation conditions for all four ports in the cycle, only the inlet and exhaust portion of the cycle are used in this study and discussed in the paper. The iteration begins at $\tau=0$ with both ends of the passage closed and the gas in a state of uniform pressure and density and velocity $(\mathrm{u}=0)$. The code then commences time integration with the opening of the exhaust port 4 (figure A1). Integration continues until the expansion fan, which was initiated by the exhaust port opening, reflects from the inlet side endwall and returns to the exhaust port. When the expansion wave reaches the exhaust port, the outflow velocity begins to drop. Eventually, it drops below zero and inflow occurs. At this point the first sub-iteration begins (figure A2). 


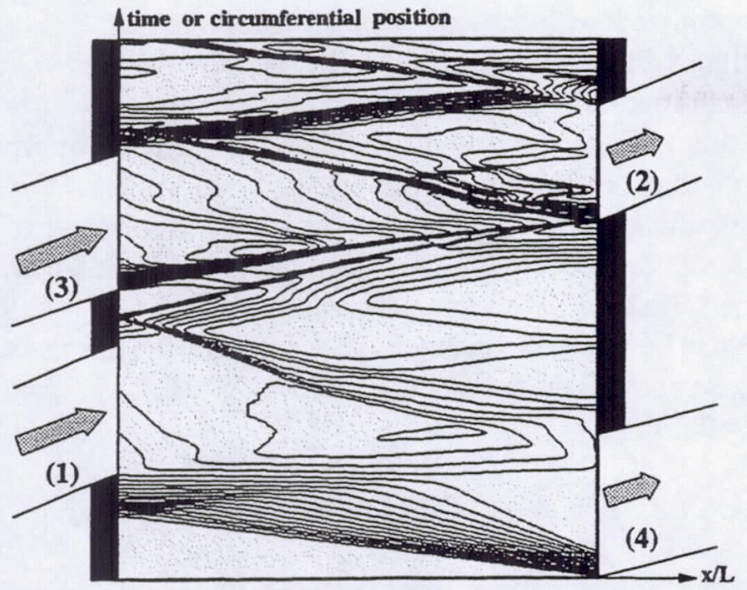

Figure 8 Pressure contours for a properly timed four port cycle

This iteration locates the exhaust port closing time such that when the closing process is complete, the port velocity has just reached zero. This closing time is then used for the remainder of the major iteration. It is noted that fixing this time is not entirely correct since the opening of the inlet port, to be described below, alters the behavior of the expansion fan and thus changes the time at which the exhaust velocity goes to zero; however, the alteration is generally very small, as is the error introduced. With the exhaust port timing set and the exhaust process complete, the exhaust mass flow and stagnation properties can be calculated. From the calculated value of $T_{4}$ and the specified value of $T_{4} / T_{1}$ the inlet temperature $T_{1}$ may be obtained. An initial guess at the inlet stagnation pressure $P_{1}$ is then made (i.e. $P_{1}=P_{4}$ ), and the iteration restarts from $\tau=0$. Integration continues until the pressure at the inlet end of the passage, adjacent to the wall, matches the inlet stagnation pressure $P_{1}$. At this point the inlet port is opened and the iteration continues until the flow in the inlet port reverses. Here, the second sub-iteration begins (figure $\mathrm{A} 3$ ). This process insures that the inlet port completes the closing process just as the shock, which has coalesced from the exhaust port 4 , reaches the inlet end of the passage. The arrival of the shock is detected by either a sudden reversal of the inlet flow or a sudden change in passage pressure adjacent to the inlet. With the inlet closing complete, the inlet mass flow is known. This is compared to the exhaust mass flow. If they match, the iteration is complete, the correct port timing is known, and the ratio $P_{4} / P_{1}$ may be calculated. If not, a new guess is made for $P_{1}$ and the calculation is restarted. Once two guesses have been made for $P_{1}$, it may subsequently be updated using

$$
\mathrm{P}_{1}^{\text {new }}=\mathrm{P}_{1}-\mathrm{e}\left[\frac{\mathrm{P}_{1}-\mathrm{P}_{1}^{\text {old }}}{\mathrm{e}-\mathrm{e}_{\text {old }}}\right]
$$

where $e$ is the difference between inlet and exhaust mass flow.

\section{OPTIMIZATION}

Optimization means finding the wave rotor geometry (i.e. L, R, $b$ and $h$ ) plus rotational speed, which gives the best performance when used as the topping cycle for a particular engine. The wave rotor is thus required to handle the mass flow of the engine, at the temperature ratio $T_{4} / T_{1}$ of the engine. It will be assumed that leakage is negligible. There are two reasons for this assumption: (1) recent experiments at NASA Lewis Research Center [10] have indicated that the effect of leakage on performance is negligible for values of the leakage parameter $\mathrm{G}$ less than 0.01 , and (2) work is in progress to develop seals to prevent, or significantly reduce, leakage. It is relatively easy to design for $\mathrm{G}<0.01$.

It will be assumed that $\dot{\mathrm{m}}, \Pi_{s}$, and $\mathrm{T}_{4} / \mathrm{T}_{1}$ are known for the engine. For most of the engines of interest, $T_{4} / T_{1}$ has a value approximately equal to 2 . The wave rotor expansion ratio giving the best performance depends on $T_{4} / T_{1}$. For $T_{4} / T_{1}$ close to 2 , calculations have shown that the optimum value of the expansion ratio is $\epsilon=0.4$. This value was assumed in the calculations following. Equating the engine mass flow to that of the wave rotor gives;

$$
\dot{\mathrm{m}}=\rho \mathrm{UA}
$$

where $\rho$ and $U$ are calculated at the wave rotor inlet port. In fact, $\rho$ and $U$ can not be calculated at the inlet port, because they are set by the conditions in the wave rotor, and are found by solving the one-dimensional CFD code. But values of $\rho$ and $U$ are required to calculate the wave rotor geometry, which must be known to evaluate input parameters for the code. This dilemma is solved either by estimating the values for $\rho$ and $U$, or using values from a previous solution, finding a solution with these values, and then using the new values from the solution to calculate new input. This is repeated until the input values are equal to the output values. The convergence is quite rapid; usually only three or four iterations are needed. Each of the $n$ inlet ports has an area given by

$$
A_{n}=\phi_{\text {in }} R h=\frac{A}{n}
$$

The value of $\phi_{\text {in }}$ comes from the cycle diagram (figure 8) for the particular value of $T_{4} / T_{1}$

$$
\phi_{\text {in }}=\frac{\tau_{\text {in }} 2 \pi}{\tau_{\text {cycle }} n}
$$

Thus, if a value of $\mathrm{R}$ is chosen, the value of $h$ is determined via

$$
h=\frac{\dot{m}}{2 \pi R \rho U}\left(\frac{\tau_{\text {cycle }}}{\tau_{\text {in }}}\right)
$$

With $h$ fixed, a value of $b$ is assumed, which fixes the hydraulic diameter from $E Q(2)$. Given $D_{h}$, and assuming a value for $L$, the friction factor (EQ (1)), and dimensionless passage opening time (EQ (3)) can be calculated. One more quantity, the relative temperature ratio, $\left(\mathrm{T}_{4} / \mathrm{T}_{1}\right)^{\text {rel }}$ is required for input into the code. The one-dimensional CFD code solves the unsteady flow within the rotor in the relative frame of the rotor. In this frame, the temperature ratio across the rotor will be different from the temperature ratio in the absolute frame. From the definition of relative stagnation temperature [12], it follows that 


$$
\begin{aligned}
& \mathrm{T}_{1}=\mathrm{T}_{1}^{\mathrm{rel}}+\frac{\mathrm{W}^{2}}{2 \mathrm{c}_{\mathrm{p}_{1}}} \\
& \mathrm{~T}_{4}=\mathrm{T}_{4}^{\mathrm{rel}}+\frac{\mathrm{W}^{2}}{2 \mathrm{c}_{\mathrm{P}_{4}}}
\end{aligned}
$$

The value of $\mathrm{W}$ depends on the rotor geometry, increasing with increasing $R$, but decreasing with increasing $L$

$$
W=\omega R=\frac{2 \pi a^{*} R}{\tau_{\text {cycle }} L n}
$$

With W known, and knowing the value of $T_{4} / T_{1}$ required, it is possible to find the value of $\left(\mathrm{T}_{4} / \mathrm{T}_{1}\right)^{\text {rel }}$. The value of $\left(\mathrm{T}_{4} / \mathrm{T}_{1}\right)^{\text {rel }}$ is used in the CFD code to solve for $\left(\mathrm{P}_{4} / \mathrm{P}_{1}\right)^{\text {rel }}$, which is then corrected to the absolute value via the isentropic relationship

$$
\frac{\frac{P_{4}}{P_{1}}}{\left(\frac{P_{4}}{P_{1}}\right)^{\text {rel }}}=\left[\frac{\frac{T_{4}}{T_{1}}}{\left(\frac{T_{4}}{T_{1}}\right)^{\text {rel }}}\right]^{\frac{\gamma}{\gamma-1}}
$$

Large values of $\mathrm{W}$ are undesireable, since then the absolute performance drops significantly.

The optimization procedure is as follows: for given values of mass flow, and absolute temperature ratio $\mathrm{T}_{4} / \mathrm{T}_{1}$, corresponding to the engine selected, values of $n, \mathrm{~L}$ and $\mathrm{R}$ are picked, thereby determining $h$. The absolute performance is calculated for various values of $b$. At small values of $b$, the opening time is small, but the friction factor is high, reducing performance. At large values of $b$, friction is low, but the large opening time reduces performance. There is an optimum value of $b$ in between, which is selected. This calculation is repeated at different values of $R$, for the same value of $L$. Then a new value of $\mathrm{L}$ is chosen, and the process repeated. The performance

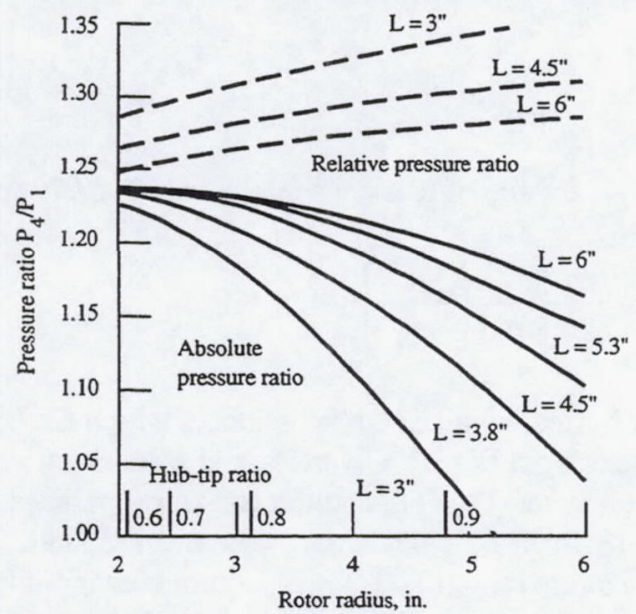

Figure 9 Relative and absolute wave rotor pressure ratio vs. length and radius for wave rotors sized for a mass flow of 4 $\mathrm{lbm} / \mathrm{s}$. The passage width has been optimized at each radius and length optimized with respect to $b$, at each value of $\mathrm{R}$, is plotted in figure 9 for $n=2$, and a mass flow of $4 \mathrm{lb} / \mathrm{sec}$. The relative performance is also shown in figure 9. At small values of $R$, the value of $\mathrm{W}$ is low, and the absolute performance approaches the relative performance, whereas at large values of $R$, there is significant difference between the two. Large values of $\mathrm{L}$ reduce $\omega$, and therefore $W$, but also increase the friction factor, reducing performance. From this plot, values of $\mathrm{L}$ and $\mathrm{R}$, and thus $b$ and $h$ can be selected which will give optimum performance. For the case shown, these values are $\mathrm{L}=5.25^{\prime \prime}, \mathrm{R}=2.5^{\prime \prime}, h=0.83^{\prime \prime}$, and $b=0.28^{\prime \prime}$.

\section{APPLICATION TO DIFFERENT ENGINES}

Using the methodology outlined above, the dimensions of optimal wave rotors can be found for gas turbine engines of various sizes. Such calculations have been made for hypothetical engines of small (4 lb/sec), medium ( $26 \mathrm{lb} / \mathrm{sec}$ ), and large (400 $\mathrm{lb} / \mathrm{sec}$ ) engines. For these engines, typical values of compression ratio and turbine inlet temperature were selected, as well as estimates of the engine dimensions. The values are listed in table 1. Also listed are the dimensions and performance of the optimal wave rotor for each engine. It will be seen that the wave rotor can be relatively small compared with the engine itself.

In one sense, there is not in fact an optimum wave rotor at all! This is because there is an optimum for each value of $n$, the number of cycles per revolution. Although the highest performance comes from $n=1$, the change in performance as $n$ is increased is very small. On the other hand, the length of the optimum rotor scales almost inversely with $\mathrm{n}$. This is illustrated

Table 1 Optimized wave rotor specifications

\begin{tabular}{llll}
\hline \hline & Small & Med. & Large \\
Engine & & & \\
Core Mass Flow (lbm/s) & 4 & 25 & 400 \\
Shaft Compression Ratio & 7 & 8 & 40 \\
Turbine Inlet Temp. (R) & 2400 & 2400 & 3200 \\
Diameter (in.) & 24 & 24 & 50 \\
Length (in.) & 40 & 48 & 190 \\
& & & \\
Wave Rotor & & & \\
Diameter (in.) & 6 & 11 & 24 \\
Length (in.) & 6 & 12 & 12 \\
Passage Height (in.) & 0.673 & 2.50 & 4.51 \\
Passage Width (in.) & 0.30 & 0.48 & 0.38 \\
Rotor Speed (r.p.m.) & 15600 & 7700 & 4200 \\
$\mathrm{~T}_{4} / \mathrm{T}_{1}$ & 2.3 & 2.3 & 1.9 \\
P $_{4} / \mathrm{P}_{1}$ & 1.232 & 1.275 & 1.204 \\
& & & \\
Performance & & & \\
untopped Spec. Pwr. (HP/lbm/s) & 121 & 151 & 276 \\
topped Spec. Pwr. (HP/lbm/s) & 149 & 183 & 300 \\
\% Increase from Wave Rotor & 23 & 21 & 8.7 \\
& & & \\
untopped SFC (lbm/HP/hr) & 0.650 & 0.529 & 0.338 \\
topped SFC (lbm/HP/hr) & 0.528 & 0.436 & 0.311 \\
\% Decrease from Wave Rotor & 18.8 & 17.5 & 8.0 \\
\hline \hline
\end{tabular}



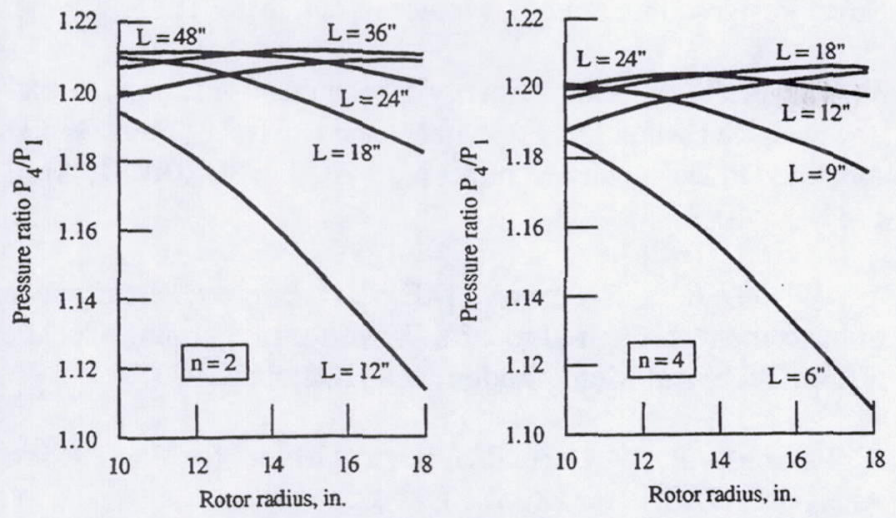

Figure 10 Absolute pressure ratio vs. length and radius for wave rotors sized for a mass flow of $400 \mathrm{lbm} / \mathrm{s}$. Curves are given for two wave rotor cycles per revolution, $(n=2)$, and for four wave rotor cycles per revolution, $(n=4)$

in figure 10 , showing performance curves for the large engine for $\mathrm{n}=2$, and $n=4$. There is almost no change in performance, but the length of the optimal $n=4$ rotor is half that of the $n=2$ rotor. From a size point of view, the $n=4$ rotor seems preferable. However, the higher value of $n$ leads to much more ducting, which could be a severe complication. In table 1 , it was assumed that $n=2$ would be appropriate for the small and medium engines, but that $n=4$ would be more appropriate for the large engine. Obviously, for a real engine, the choice of $n$ will be made by the engine designer based on engineering considerations. Another complication is indicated in figure 9, in which approximate values of the wave rotor hub-tip ratio are given. Performance optimization for the small engine drives the choice of wave rotor radius to small values, which results in low values of the hub-tip ratio. The one-dimensional code does not presently account for hub-tip ratio effects. Consequently, the lower limit of hub-tip ratio for a wave rotor is not known. Modification of the code is required to include this effect. Turbomachinery typically has a lower limit of hub-tip ratio of 0.7 . It seems reasonable to use a similar criterion for wave rotors also, and this may limit the value of radius used.

The calculated performance of the three optimal wave rotors has been plotted in figure 11. It is seen that each wave rotor is predicted to give performance better than that of the G.E. wave rotor. This is reflected in the wave rotor enhanced performance of the three hypothetical engines, as listed in table 1. All three engines will give better performance than that indicated by the calculation based on the GE wave rotor performance, increasing the specific power another $2.3 \%$ for the small engine, $3.1 \%$ for the medium engine, and $2.7 \%$ for the large engine, and reducing the specific fuel consumption a further $1.6 \%$ for the small engine, $2.6 \%$ for the medium engine, and $2.4 \%$ for the large engine. The increase in specific power, and reduction in specific fuel consumption that can be expected for engines corresponding to the engine design line of figure 6 , when enhanced by wave rotors optimized for the small and large engines is indicated in figure 12.

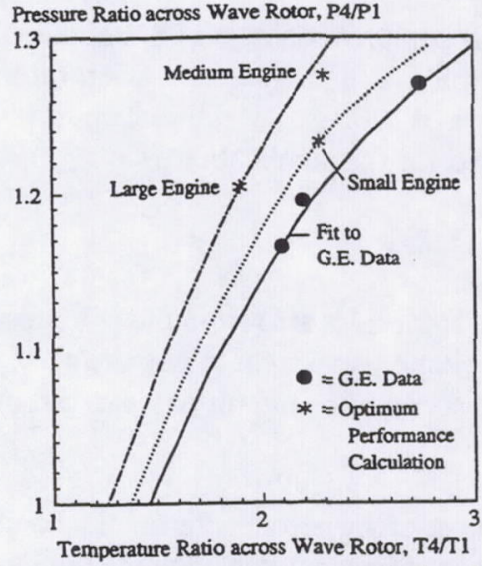

Figure 11 Performance of wave rotors optimized for the small, medium, and large engines together with the performance of the G.E. wave rotor measured by Klapproth et al. [1]

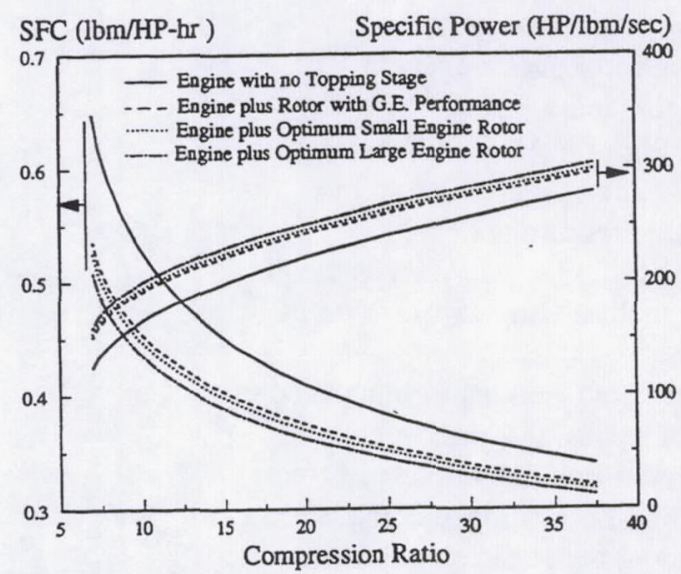

Figure 12 The improvement in gas turbine engine performance that can be obtained with a wave rotor topping cycle employing wave rotors of differing performance, for engines corresponding to the engine design line

\section{SUMMARY AND CONCLUSIONS}

The advantages of using a wave rotor topping cycle on a gas turbine engine have been reviewed. A one-dimensional, experimentally validated, CFD model for calculation of wave rotor performance has been described briefly. This model was used to find optimally sized wave rotors for three hypothetical engines. It is concluded that use of a wave rotor topping cycle on a gas turbine engine can: (1) Based on demonstrated wave rotor performance, give increases in specific power of $19.2 \%$ at a compression ratio of 8 , turbine inlet temperature of $2400 \mathrm{R}$, and $5.8 \%$ at a compression ratio of 40 , turbine inlet temperature of 3200 R. (2) Based on demonstrated wave rotor performance, give reductions in specific fuel consumption of $16.2 \%$ at a compression ratio of 8 , turbine inlet temperature of $2400 \mathrm{R}$, and $5.6 \%$ at a compression ratio of 40 , turbine inlet temperature of $3200 \mathrm{R}$. (3) With optimally sized wave rotors, give increases in specific power of $21 \%$ at a compression ratio of 8 , turbine inlet temperature of $2400 \mathrm{R}$, and $8.7 \%$ at a compression ratio of 40 , turbine inlet temperature of $3200 \mathrm{R}$. (4) With optimally sized 
wave rotors, give reductions in specific fuel consumption of $17.5 \%$ at a shaft compression ratio of 8 , turbine inlet temperature of $2400 \mathrm{R}$, and $8 \%$ at a shaft compression ratio of 40 , turbine inlet temperature of $3200 \mathrm{R}$. In addition, optimal wave rotors are reasonably sized for this application.

\section{NOMENCLATURE}

$A=$ total wave rotor inlet area from the compressor

$A_{n}=$ area of a single wave rotor inlet $(=A / n)$

$a^{*}=$ reference speed of sound (in passage just prior to opening to exhaust)

$\mathrm{b}=$ wave rotor passage width

$c_{p 1}=$ specific heat of air before entering the combustor

$\mathrm{c}_{\mathrm{p} 4}=$ specific heat of gas after leaving the combustor

$\mathrm{D}_{\mathrm{h}}=$ passage hydraulic diameter

$\mathrm{e}=$ difference between inlet and exhaust mass flow rate used in EQ (4)

$\mathrm{G}=$ leakage parameter $(=2 \delta / \mathrm{h})$

$\mathrm{h}=$ wave rotor passage height

$\mathrm{L}=$ wave rotor passage length

$\dot{\mathrm{m}}=$ engine mass flow

$\mathrm{n}=$ number of cycles on the wave rotor

$\mathrm{P}_{\mathrm{i}}=$ stagnation pressure at port $\mathrm{i}$

$\mathrm{p}_{\mathrm{i}}=$ static pressure at port $\mathrm{i}$

$\mathrm{R}=$ rotor radius at mid-passage height

$T_{i}=$ stagnation temperature at port $i$

$\mathrm{t}=$ time

$\mathrm{U}=$ axial flow velocity at input to rotor

$\mathrm{u}=$ velocity inside a passage

$\mathrm{W}=$ circumferential velocity of rotor

$\gamma=$ specific heat ratio for combustion gases

$\delta=$ spacing between rotor and end-wall

$\epsilon=$ expansion ratio, ratio of $\mathrm{p}_{4}$ to pressure in passage before opening to the exhaust port 4

$\mu=$ viscosity

$\rho=$ density at input to rotor

$\rho^{*}=$ reference density, i.e. density in passage just prior to opening to exhaust port 4

$\Pi_{s}=$ shaft compression ratio, i.e. $\mathrm{P}_{1} / \mathrm{P}_{0}$, which is the compression ratio of the mechanical compressor

$\tau=$ non-dimensional time $=\mathrm{t} \mathrm{a}^{*} / \mathrm{L}$

$\tau_{\text {cycle }}=$ non-dimensional time for one cycle

$\tau_{\text {in }}=$ non-dimensional time inlet port is open

$\tau_{\mathrm{ot}}=$ non-dimensional passage opening time

$\phi_{\text {in }}=$ angle subtended by inlet port

$\omega=$ rotor angular velocity (radians/sec)

subscripts $1,2,3,4=$ wave rotor ports indicated in figure 1

\section{REFERENCES}

1. Klapproth, J.F., Perugi, A., Gruszczynski, J.S., Stoffer, L.J., and Alsworth, C.C. "A Brief Review of the G.E. Wave Rotor Program (1958 - 1963)", reported by Mathur, A. in Proceedings of the 1985 ONR/NAVAIR Wave Rotor Research and Technology Workshop, Report NPS-67-85-008, Naval Postgraduate School, Monterey, CA, May 1985.

2. Moritz, R., "Rolls-Royce Study of Wave Rotors 1965-1970", in Proceedings of the 1985 ONR/NAVAIR Wave Rotor Research and Technology Workshop, Report NPS-67-85-008, Naval Postgraduate School, Monterey, CA, May 1985.

3. Thayer, W.J. et al. "Energy Exchanger Performance and Power Cycle Evaluation Experiment and Analysis", Final Report submitted to DoE under Contract No. AC06-78ER01084, April 1981.

4. Wilson, J., and Paxson, D.E. "Jet Engine Performance Enhancement through Use of a Wave-Rotor Topping Cycle", NASA Technical Memorandum 4486, October 1993.

5. Paxson, D.E. "A General Numerical Model for Wave Rotor Analysis", NASA TM 105740, 1992.

6. Paxson, D.E. "A Comparison between Numerically Modelled and Experimentally Measured Loss Mechanisms in Wave Rotors", presented at the 29th Joint Propulsion Conference, Monterey, California, paper No. AIAA-93-2522.

7. Paxson, D.E. and Wilson, Jack "Recent Improvements to and Validation of the One Dimensional NASA Wave Rotor Model", NASA Technical Memorandum 106913, 1995.

8. Kentfield, J. A. C., "An Examination of the Performance of Pressure Exchanger Equalizers and Dividers", Journal of Basic Engineering, p361 September, 1969.

9. Mattingly, J.D., Heiser, W.H., and Daley, D.H. "Aircraft Engine Design", A.I.A.A., New York, 1987.

10. "Specifications, U.S. Gas Turbine Engines", Aviation Week and Space Technology, p165 March 19, 1990.

11. Wilson, J. and Fronek, D. "Initial Results from the NASA-Lewis Wave Rotor Experiment", presented at the 29th Joint Propulsion Conference, Monterey, California, 1993, paper No. AIAA-93-2521.

12. Glassman, A. J. Editor, "Turbine Design and Application", NASA SP-290, 1994 
APPENDIX A: CFD ITERATION FLOWCHARTS

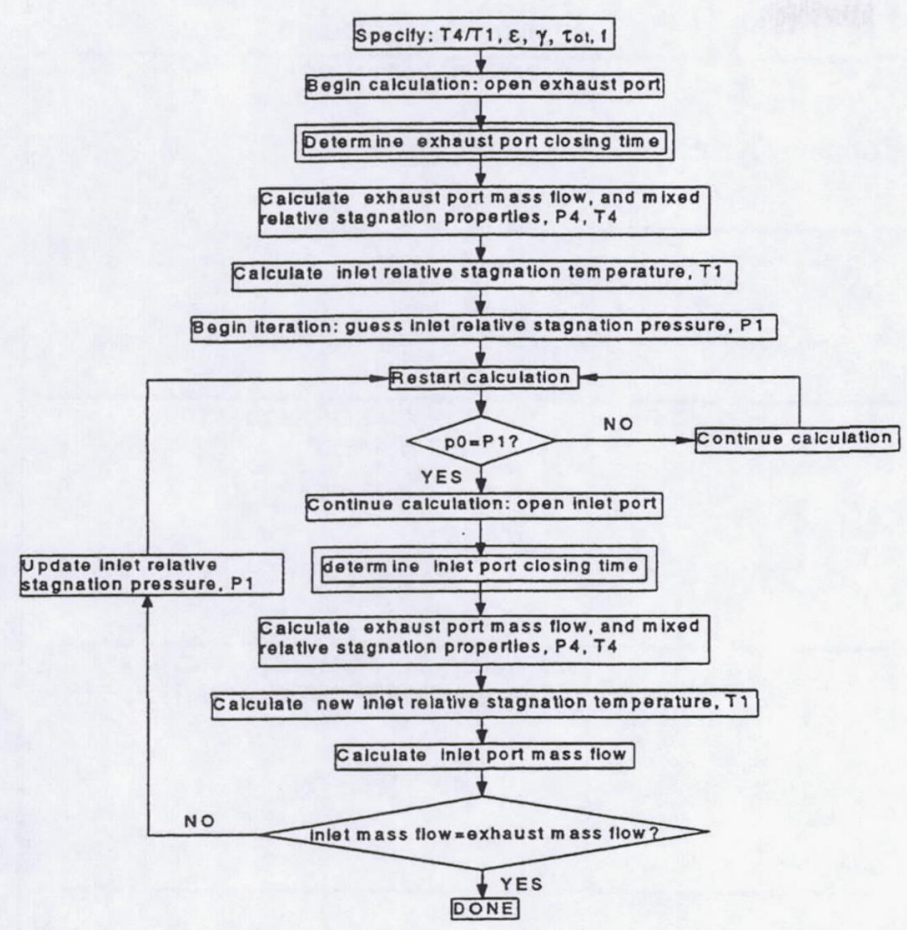

Figure A1 Four port iteration flow chart

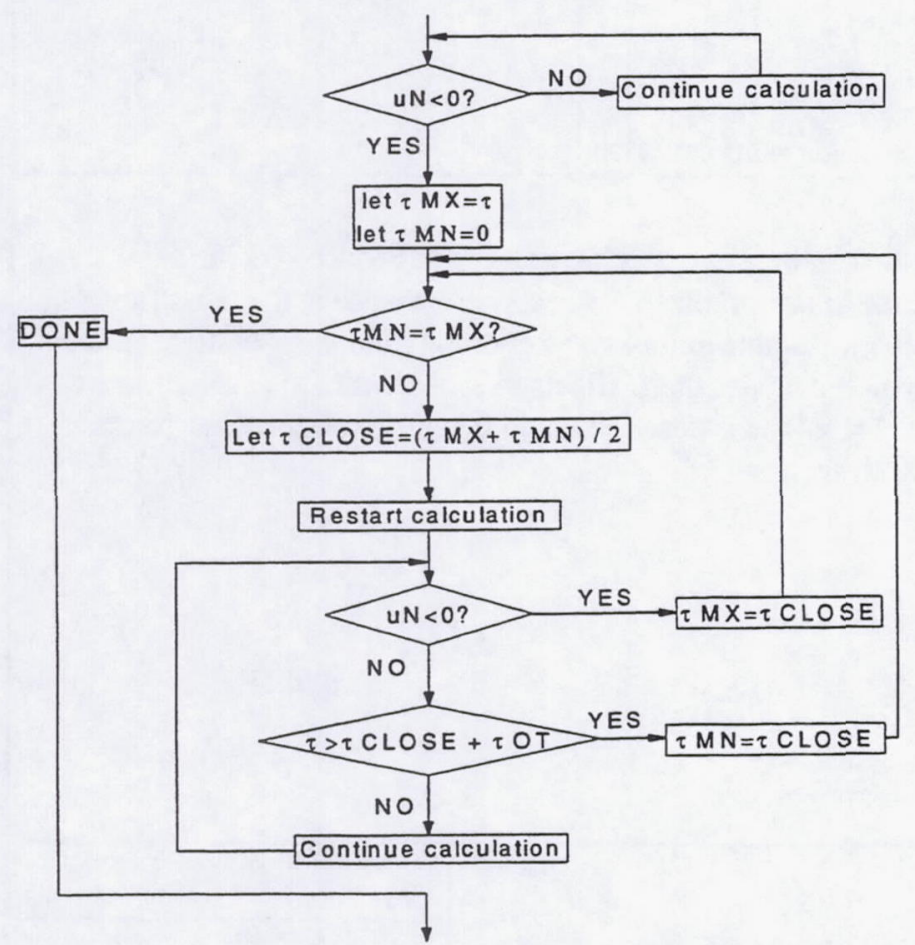

Figure A2 Exhaust port closing sub-iteration flow chart

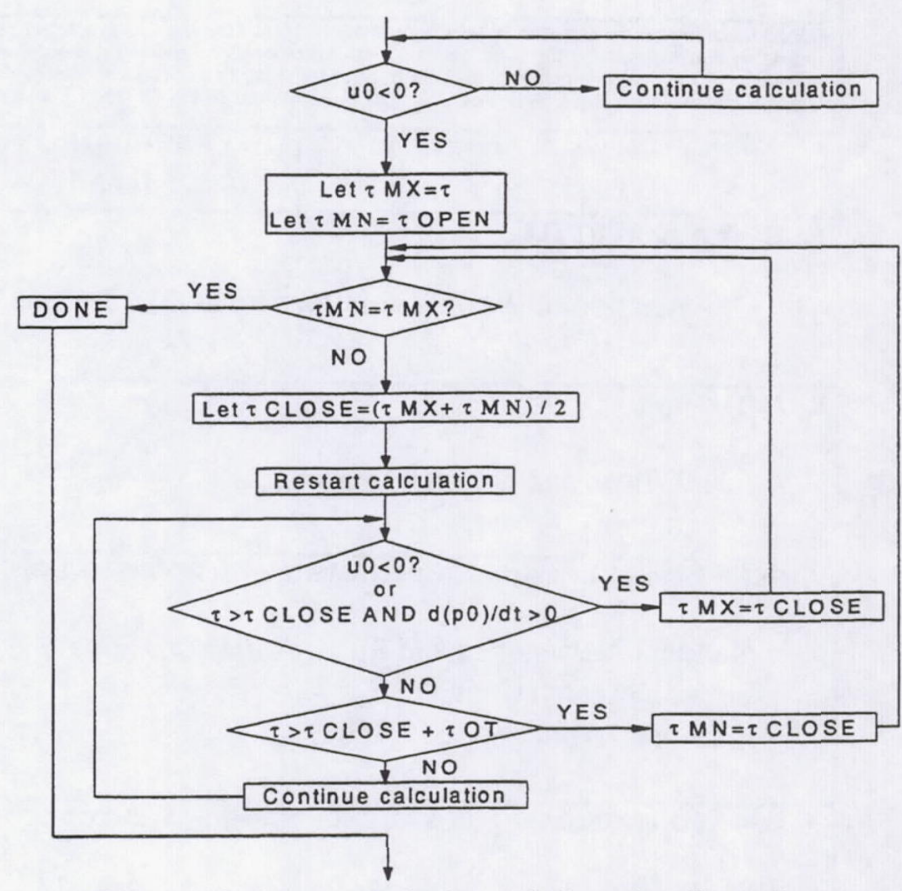

Figure A3 Inlet port closing sub-iteration flow chart 


\section{REPORT DOCUMENTATION PAGE}

Public reporting burden for this collection of information is estimated to average 1 hour per response, including the time for reviewing instructions, searching existing data sources, gathering and maintaining the data needed, and completing and reviewing the collection of information. Send comments regarding this burden estimate or any other aspect of this collection of information, including suggestions for reducing this burden, to Washington Headquarters Services, Directorate for lion Project (0704-0188), Washington, DC 20503.

\begin{tabular}{l|l|l|l}
\hline Davis Highway, & 3. REPORT TYPE AND DATES COVERED
\end{tabular}

\begin{tabular}{|l|c|c|}
\hline 1. AGENCY USE ONLY (Leave blank) & $\begin{array}{r}\text { 2. REPORT DATE } \\
\text { June } 1995\end{array}$ & $\begin{array}{r}\text { 3. REPORT TYPE AND DATES COVERED } \\
\text { Technical Memorandum }\end{array}$ \\
\hline
\end{tabular}

\section{TITLE AND SUBTITLE}

5. FUNDING NUMBERS

Optimization of Wave Rotors for Use as Gas Turbine Engine Topping Cycles

6. $\operatorname{AUTHOR}(\mathrm{S})$

WU-505-90-58

Jack Wilson and Daniel E. Paxson

7. PERFORMING ORGANIZATION NAME(S) AND ADDRESS(ES)

National Aeronautics and Space Administration

Lewis Research Center

Cleveland, Ohio 44135-3191

8. PERFORMING ORGANIZATION REPORT NUMBER

E-9689

9. SPONSORING/MONITORING AGENCY NAME(S) AND ADDRESS(ES)

10. SPONSORING/MONITORING AGENCY REPORT NUMBER

National Aeronautics and Space Administration

Washington, D.C. 20546-0001

NASA TM-106951

11. SUPPLEMENTARY NOTES

Prepared for 1995 Aerospace Atlantic Conference and Exposition sponsored by the Society of Automotive Engineers, Dayton, Ohio, May 23-25, 1995. Jack Wilson, NYMA, Inc., Engineering Services Division, 2001 Aerospace Parkway, Brook Park, Ohio 44142 (work funded by NASA Contract NAS3-27186), and Daniel E. Paxson, NASA Lewis Research Center. Responsible person, Daniel E. Paxson, organization code 2560, (216) 433-8334.

12a. DISTRIBUTION/AVAILABILITY STATEMENT 12b. DISTRIBUTION CODE

Unclassified - Unlimited

Subject Category 07

This publication is available from the NASA Center for Aerospace Information, (301) 621-0390.

\section{ABSTRACT (Maximum 200 words)}

Use of a wave rotor as a topping cycle for a gas turbine engine can improve specific power and reduce specific fuel consumption. Maximum improvement requires the wave rotor to be optimized for best performance at the mass flow of the engine. The optimization is a trade-off between losses due to friction and passage opening time, and rotational effects. An experimentally validated, one-dimensional CFD code, which includes these effects, has been used to calculate wave rotor performance, and find the optimum configuration. The technique is described, and results given for wave rotors sized for engines with sea level mass flows of 4,26 , and $400 \mathrm{lb} / \mathrm{sec}$.

14. SUBJECT TERMS

Wave rotor; Optimization; Jet engine topping cycle

17. SECURITY CLASSIFICATION OF REPORT

Unclassified
18. SECURTYY CLASSIFICATION OF THIS PAGE

Unclassified
19. SECURITY CLASSIFICATION OF ABSTRACT Unclassified
5. NUMBER OF PAGES

11

16. PRICE CODE

$\mathrm{A} 03$

20. LIMITATION OF ABSTRACT

Standard Form 298 (Rev. 2-89) Prescribed by ANSI Std. Z39-18 298-102 